\title{
First randomized study
}

Children with acute myeloid leukaemia (AML) achieve long-term survival in $60 \%$ of cases. However, in patients who relapse

following treatment, the odds are not as far in their favour-the probability of overall survival is $16-34 \%$. Although a number of treatment options are available for patients that relapse, no randomized clinical studies have been conducted to determine which treatment is optimal. Now, a study has shown that the addition of an anthracyline to an induction regimen is well tolerated and improved early treatment response.

The international clinical trial was led by Gertjan Kaspers and recruited 568 patients under 21 years of age with relapsed or refractory AML. Of these patients, 394 were randomly assigned to receive reinduction therapy with either fludarabine, cytarabine and granulocyte colony-stimulating factor (FLAG) or to FLAG in combination with liposomal daunorubicin. Kaspers explains why the study agent was selected: "liposomal anthracyclines are known to be less cardiotoxic in general, and liposomal daunorubicin is better for leukaemias because of its myelosuppression."

Kaspers and his colleagues selected the status of the bone marrow

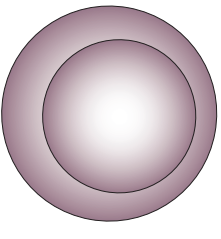
on day 28 (directly before the second course of chemotherapy) as the primary end point of the trial. As he points out, this end point is "less conventional, but known to be highly prognostic in paediatric relapsed AML and does not require prolonged follow up." The bone marrow status at day 28 for both patient groups was good (defined as $\leq 20 \%$ leukaemic blasts) in $80 \%$ of the patients assigned to receive the FLAG plus liposomal daunorubicin combination and $70 \%$ of the patients receiving FLAG alone. This difference was significant, but did not translate into improved overall survival.

Despite this seemingly disappointing outcome, Kaspers is pleased with the results of the trial: "an approach strictly working according to protocol led to a very significantly improved survival [compared with previously reported studies] of $36 \%$ for the nearly 600 patients registered." In addition, the improved early response as determined by the bone marrow status is also a cause for optimism: "to consolidate these better early responses, further improvements in subsequent therapy are needed. However, the first step-a good initial response-is important."

Crucially, the study was able to demonstrate that the toxicity of the two regimens was essentially the same (although long-term cardiotoxicity data are not yet available). This demonstration of an acceptable safety profile, alongside the improved complete remission rate in the combination arm, will enable future studies to use liposomal anthracylines as part of the core therapy backbone to assess novel targeted agents. Furthermore, the results from this trial show that large-scale trials in these patients are possible and informative.

\section{Rebecca Kirk}

Original article Kaspers, G. J. L. et al. Improved outcome in pediatric relapsed acute myeloid leukemia: results of a randomized trial on liposomal daunorubicin by the international BFM study group. J. Clin. Oncol. doi:10.1200/ JC0.2012.43.7384 